\title{
Mozambique : la réforme du système d'éducation professionnelle
}

Pierre Homerin

\section{OpenEdition}

1 Journals

Édition électronique

URL : https://journals.openedition.org/ries/6102

DOI : 10.4000/ries.6102

ISSN : 2261-4265

Éditeur

France Education international

\section{Édition imprimée}

Date de publication : 30 avril 2018

Pagination : 15-17

ISBN : 978-2-85420-618-0

ISSN : 1254-4590

Référence électronique

Pierre Homerin, « Mozambique : la réforme du système d'éducation professionnelle », Revue internationale d'éducation de Sèvres [En ligne], 77 | avril 2018, mis en ligne le 30 avril 2020, consulté le 25 juin 2021. URL : http://journals.openedition.org/ries/6102 ; DOI : https://doi.org/10.4000/ries.6102

Ce document a été généré automatiquement le 25 juin 2021.

(c) Tous droits réservés 


\title{
Mozambique : la réforme du système d'éducation professionnelle
}

\author{
Pierre Homerin
}

Deuxième pays lusophone au monde, avec une population de près de 30 millions d'habitants en 2016, pour une superficie de $801590 \mathrm{~km}^{2}$, le Mozambique se situe au croisement des influences africaines, arabes, asiatiques, sud-américaines et européennes. Ouvert sur l'Océan indien, le pays fait partie de la Communauté de développement de l'Afrique australe et appartient à la Communauté des pays de langue portugaise depuis 1996 mais il est aussi devenu membre du Commonwealth en 1995 et membre observateur de l'Organisation internationale de la Francophonie (OIF) depuis 2006.

2 Depuis son indépendance, en 1975, le Mozambique a adopté la langue portugaise comme unique langue officielle: alors que plus de $80 \%$ de la population urbaine s'exprime en portugais, les onze provinces regroupent plus d'une quarantaine d'ethnies et de langues «nationales » d'origine bantoue, dont seize sont enseignées de façon encore expérimentale dans le système scolaire.

3 Encore $181^{\mathrm{e}}$ sur 189 pays à l'indice de développement humain en 2015 (PNUD), avec une espérance de vie de 55 ans (Banque mondiale), le Mozambique a été l'un des pays les plus pauvres et aidés par la coopération internationale depuis 1992, date de la fin des seize années de guerre civile qui ont ruiné le pays ${ }^{1}$.

4 Les premières décennies ont d'abord été consacrées à la reconstruction de l'État mozambicain et au développement des infrastructures, avec, pour l'éducation, une priorité à l'enseignement primaire : en 1997, 66,87 \% des enfants étaient scolarisés dans le primaire, $6,9 \%$ dans le secondaire, et $0,3 \%$ dans le supérieur.

5 Tous les bailleurs y ont opéré, simultanément ou successivement, sur un héritage colonial portugais stratifié par l'influence soviétique et est-allemande des années 1980, et ont ainsi modelé une culture d'adaptation passagère à l'aide internationale, favorable au développement de projets éducatifs, mais sans continuité ni reprise nationale, les bailleurs utilisant les mêmes rares ressources humaines nationales pour de nouveaux projets toujours acceptés par un pays aux multiples carences. 

éducatif : après quelques projets sectoriels, une impulsion nationale a enfin été donnée avec le Programme stratégique de développement du secteur de l'éducation financé par douze bailleurs ${ }^{2}$ unifiés.

7 La réforme de l'enseignement de base de 2004 met la priorité sur l'accès à l'éducation, met en œuvre la généralisation de la gratuité de l'enseignement et l'introduction de nouveaux programmes, avec la fourniture des manuels scolaires. L'effet se fait tout de suite sentir : le taux de scolarisation à 6 ans passe de $30 \%$ en 2000 à $73 \%$ en 2008 et $93 \%$ en 2016. En 2004, 3,69 millions d'enfants étaient scolarisés dans le primaire, pour 5,45 millions en 2011 et 6,7 millions en 2014 (Unicef); 1450 salles de classe sont construites par an: l'enseignement primaire passe de 7500 écoles en 2000 à plus de 17000 en 2014, et l'enseignement secondaire d'une centaine en 2000 à près d'un millier actuellement ${ }^{3}$.

8 Malgré ces progrès impressionnants, beaucoup de défis doivent encore être relevés : selon l'Unesco, 1,2 million d'enfants, soit $23 \%$, restent encore en dehors du système éducatif et la qualité de l'enseignement n'est toujours pas au rendez-vous : si le taux de conclusion de l'enseignement primaire passe de $16 \%$ en 2000 à $42 \%$ en 2006, il stagne depuis à $47 \%$ et plus de la moitié des enfants ne termine toujours pas l'enseignement primaire.

9 Aussi le nouveau Plan stratégique pour l'éducation 2012-2016, prolongé jusqu'en 2019, a-t-il mis l'accent sur l'augmentation d'une offre de qualité par une meilleure formation des ressources humaines et le renforcement des liens entre le système éducatif et les besoins du marché par le biais d'un enseignement technique et professionnel de qualité et l'appropriation de l'école au niveau local.

\section{Le Programme intégré pour la réforme de l'éducation professionnelle (PIREP)}

En 2006, une ambitieuse réforme de l'éducation professionnelle a été lancée, soutenue par des financements de la Banque mondiale et d'un groupe de bailleurs internationaux : le Programme intégré pour la réforme de l'éducation professionnelle (PIREP), caractérisé par l'implantation d'un nouveau concept unique, l'éducation professionnelle (EP), qui réunit les deux composantes traditionnelles antérieures :

- l'enseignement technique et professionnel géré par la direction nationale de l'enseignement technique du ministère de l'éducation ;

- la formation professionnelle, sous la responsabilité de l'Institut national de la formation professionnelle (INFP), rattaché au ministère du Travail.

11 Le PIREP, mis en œuvre sur quinze années, vise la transformation de l'éducation professionnelle en un système fondé sur la demande et les besoins, et non plus sur une offre de formation. Construit à partir d'un Cadre national de qualifications développé avec la participation des employeurs et des partenaires sociaux, il s'articule autour de trois axes :

- amélioration de la coordination entre enseignement professionnel et enseignement supérieur, par le développement de cursus fondés sur l'approche par compétences;

- amélioration de la coordination entre les institutions d'enseignement secondaire ;

- création à terme d'une nouvelle Autorité nationale pour l'éducation professionnelle.

Revue internationale d'éducation de Sèvres, 77 | avril 2018 
12 Le système national de qualifications professionnelles ainsi conçu part donc de l'identification des besoins de formation pour concevoir des curricula (dénommés "qualifications ») découpés en unités de compétences ou modules validés puis classés dans le Cadre national de qualifications sur cinq niveaux, qui correspondent chacun à un «certificat professionnel». Parallèlement, ces unités de compétence seront constituées en système de crédits permettant, à terme, l'instauration de procédures de reconnaissance des acquis de l'expérience. Les deux premiers niveaux recouvrent des compétences de base et un degré d'autonomie qui ne correspondent pas encore forcément à un emploi spécifique, mais à des compétences nécessaires, et non suffisantes, à l'exercice d'un métier.

Les trois autres niveaux correspondent de fait essentiellement à l'enseignement secondaire technique professionnel et, domaine après domaine, les cursus ont été réécrits et construits en unités de compétence afin de les introduire dans le Cadre national et les mettre tous en fonctionnement à l'horizon 2018.

15 À terme, ce Cadre national de qualifications devra être harmonisé avec son équivalent sud-africain, dans la perspective de la construction d'un Cadre régional de qualifications commun pour la Communauté de développement de l'Afrique australe.

16 L'une des caractéristiques les plus importantes du PIREP est la mise en place d'outils de garantie de la qualité de l'éducation professionnelle: élaboration partenariale des qualifications, accréditation des établissements, formation spécifique des enseignants, évaluation des compétences des élèves, mise en place d'un corps de "vérificateurs" internes et externes, du processus d'évaluation des compétences, et certification pour chaque acteur du processus, élève, enseignant, vérificateur et établissement.

17 La première étape (2006-2011) a donc consisté à construire le canevas du Cadre national inspiré du Scottish Credit and Qualifications Framework, à partir du relevé des formations existantes et des besoins exprimés notamment par les employeurs, et à fixer les niveaux de qualification avec leur progression, leurs critères d'entrée et de sortie.

Afin de garantir un niveau constant de qualité, il a fallu concevoir et mettre en place un système d'outils et de procédures liés à l'approche par compétences: manuel de développement des qualifications et modules curriculaires, orientations méthodologiques, manuel de validation et d'enregistrement des qualifications, manuels de l'évaluateur, du vérificateur interne, du vérificateur externe.

19 La Loi du 23 septembre 2014, revue et republiée le 16 juin 2016, est venue structurer la totalité du secteur, définissant et caractérisant concepts, structures, dispositifs, établissements et acteurs et validant le Cadre national de qualifications comme structure globale de l'éducation professionnelle.

20 La gouvernance du système a été confiée à la nouvelle Autorité pour l'éducation professionnelle, créée par décret du 7 novembre 2016, qui devra gérer la totalité du système à l'horizon 2018, après une année 2017 de mise en place, de recrutements et d'élaboration de procédures. Elle aura pour charge d'émettre les certificats, les autorisations et licences de fonctionnement ainsi que l'ensemble des diplômes, dans une perspective de garantie de la qualité généralisée à tous les acteurs et établissements.

21 La construction d'un cadre national de qualifications et la création d'une Autorité nationale a permis de rendre les Mozambicains propriétaires de leur avenir et de 
s'insérer dans une politique régionale de développement économique maitrisée. Mais, chargée de mettre en œuvre la réforme au niveau national, l'ANEP aura-t-elle les moyens de le faire? Un dispositif de financement est prévu, le Fonds national de l'éducation professionnelle, créé en juin 2017, qui sera abondé par une contribution des employeurs s'ajoutant à l'aide de l'État, mais celui-ci sera-t-il suffisant?

De fait, la réforme dépend toujours des financements d'infrastructures, d'équipements ou d'élaboration de curricula effectués par des bailleurs nationaux ou internationaux, liés à leurs intérêts et priorités.

\section{NOTES}

1. On estime à plus de $60 \%$ la destruction du réseau des écoles élémentaires (Banque mondiale).

2. Parmi lesquels la Banque mondiale, «la Banque africaine» de développement, l'Union européenne et les coopérations portugaise, espagnole, danoise, irlandaise, française, canadienne et japonaise.

3. «Exame nacional 2015 da Educação para Todos, Moçambique ». Contact : efa2015reviews@unesco.org

\section{INDEX}

Mots-clés : enseignement professionnel, réforme de l'enseignement

Keywords : vocational education, educational reform

Palabras claves : enseñanza profesional, reforma de la educación

Index géographique : Mozambique

\section{AUTEUR}

\section{PIERRE HOMERIN}

Pierre Homerin est chargé de programmes au Centre international d'études pédagogiques (CIEP, France) et diplômé ès lettres de l'Université de Paris-X Nanterre. Une grande partie de sa carrière s'est déroulée dans les pays lusophones : enseignant au Brésil, attaché de coopération pour le français au Portugal, chef de projet au Mozambique, attaché de coopération éducative en Angola. Conseiller en formation continue en France et directeur régional du SENAI au Brésil, il a développé une expertise en formation professionnelle et mène actuellement le projet de mise en place du Système national de certifications au Mozambique pour la Banque mondiale. Courriel : homerin@ciep.fr 